Research Article

\title{
Plasma-Derived Exosomal microRNA-130a Serves as a Noninvasive Biomarker for Diagnosis and Prognosis of Oral Squamous Cell Carcinoma
}

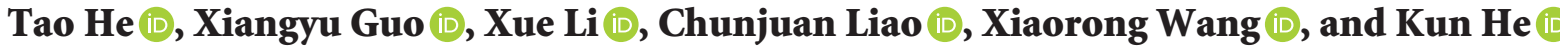 \\ State Key Laboratory of Oral Diseases \& National Clinical Research Center for Oral Diseases, \\ Department of Oral and Maxillofacial Surgery, West China Hospital of Stomatology, Sichuan University, Chengdu 610000, China
}

Correspondence should be addressed to Kun He; kunhe1982@163.com

Received 12 January 2021; Revised 19 March 2021; Accepted 7 April 2021; Published 17 April 2021

Academic Editor: Dali Zheng

Copyright ( 2021 Tao He et al. This is an open access article distributed under the Creative Commons Attribution License, which permits unrestricted use, distribution, and reproduction in any medium, provided the original work is properly cited.

Exosomal microRNAs (miRNAs) are considered as potential stable biomarkers in many types of human cancer, but investigations of plasma-derived exosomal miRNAs in oral squamous cell carcinoma (OSCC) are still lacking. The aim of this study is to evaluate the diagnostic and prognostic values of exosomal miR-130a in OSCC patients. Exosomes were isolated from plasma samples which were collected from 184 OSCC patients before surgery and 196 healthy individuals. Primary OSCC and paired adjacent noncancerous tissues were also obtained from 47 OSCC patients. The expression levels of miR-130a were analyzed by quantitative real-time PCR (qRT-PCR). Our results showed that the expression levels of exosomal miR-130a were significantly higher in OSCC patients than those of the healthy controls $(p<0.0001)$. Also, the expression of miR-130a was also significantly upregulated in OSCC tissues compared with paired adjacent noncancerous tissues $(p<0.0001)$. A significant positive correlation was found between exosomal miR-130a and tissue miR-130a levels. Receiver operating characteristic (ROC) analyses yielded an AUC value of 0.812 in discriminating OSCC patients from healthy controls. Furthermore, high levels of exosomal miR-130a were associated with the late T-stage $(p=0.024)$, advanced TNM stage $(p=0.003)$, and poorly differentiated OSCC $(p=0.013)$. Patients with high exosomal miR-130a expression had significantly worse 3-year overall survival (OS) and recurrence-free survival (RFS). Multivariate analysis indicated that exosomal miR-130a was an independent prognostic factor for OS $(p=0.001)$ and RFS $(p=0.003)$. Our results suggest that exosomal miR-130a may serve as a promising diagnostic and prognostic biomarker for OSCC patients.

\section{Introduction}

According to the latest cancer statistics, oral cancer is the leading cause of cancer-related morbidity among men, with high frequency in certain regions or specific countries, such as India and Sri Lanka [1]. In China, it is estimated that there are approximately 48,100 new cases diagnosed as oral cancer and 22,100 patients died of this malignancy in 2015 [2]. Over $90 \%$ of all oral cancers are classified as oral squamous cell carcinoma (OSCC) [3]. Although the therapeutic strategies have been greatly improved, the 5-year overall survival rate of OSCC patients is still less than $50 \%$, which is mainly because of the high rates of the local recurrence or distant metastasis after primary treatment [4]. A histological examination on tissue biopsy of suspicious lesions is considered as the gold standard for OSCC diagnosis, but it is invasive, costly, and potentially harmful. Thus, most of OSCC cases are detected at advanced stages, which could lead to poor prognosis $[3,5]$. Therefore, a novel diagnostic or prognostic biomarker which is less invasive and cost effective is urgently needed in clinical practice, and this could definitely improve patient survival.

Exosomes are nanometer-sized $(30-150 \mathrm{~nm})$ vesicles which are secreted by multivesicular bodies (MVBs). The main components of exosomes' external part are lipids, while various molecules such as proteins, mRNAs, microRNAs (miRNAs), genomic DNA, and mitochondrial DNA (mtDNA) are found inside [6, 7]. Tumor cells are known to secrete large amounts of exosomes which are at least 10-fold more than that of the normal cells and widely present in 
various body fluids, such as urine, saliva, blood, lymph, and cerebrospinal fluid $[8,9]$. Exosomes could act as a delivery system which is important for communication between cells [10]. Therefore, exosomes are considered as an important component of the tumor microenvironment, which may contribute to tumor invasion and metastasis through communication with the surrounding stromal tissue or distant organs [11, 12]. MicroRNAs (miRNAs) are short (18-25 nucleotides long), single-stranded, noncoding RNAs which can regulate gene expression through inhibition of translation or degradation of the targeted mRNA [13]. As we all know, miRNAs have been proved to play critical roles in multiple cellular process of cancer, such as differentiation, proliferation, apoptosis, migration, and invasion [14]. Exosomes could envelop specific miRNAs and maintain its integrity in circulation, and exosomal miRNAs could possibly interfere with tumor microenvironment and facilitate tumor growth, invasion, and metastasis through taken up by surrounding or distant cells $[15,16]$. Therefore, circulating exosomal miRNAs hold great promise to be used as novel noninvasive biomarkers in cancer diagnosis and prognosis.

Recently, accumulating evidences have confirmed that exosomal miRNAs are potential biomarkers for cancer diagnosis and prognosis, including lung cancer [17], liver cancer [18], colorectal cancer [19], and breast cancer [20]. In OSCC, only few studies have explored the association between the exosomal miRNAs and tumor growth, invasion, and metastasis, such as miR-24-3p and miR-382-5p [21, 22]. As a member of the microRNA-130 family, miR-130a has been extensively studied in various types of human cancer. However, the results are inconsistent and disputable. Several studies have demonstrated that miR-130a serves as a tumor suppressor in liver cancer and prostate cancer [23,24], while miR-130a has also been reported to exhibit tumorigenic characteristics in breast cancer, esophageal cancer and cervical cancer [25-27]. Moreover, the diagnostic and prognostic value of miR-130a in OSCC is still unknown. In the present study, we aim to investigate the correlation between plasma-derived exosomal miR-130a and clinicopathological characteristics of OSCC and the potential of using exosomal miR-130a as a biomarker for diagnosis and prognosis in OSCC patients.

\section{Material and Methods}

2.1. Clinical Samples. The present study was approved by the ethics committee of West China Hospital. A total of 184 patients with OSCC who underwent surgical resection at our hospital and 196 age and sex matched healthy controls were recruited between 2014 and 2016. None of the OSCC patients received radiotherapy or chemotherapy before surgery. All tumors were staged according to the seventh edition of the American Joint Committee on Cancer system. Blood samples were collected in EDTA tubes from all OSCC patients before surgery and healthy individuals. For plasma, fresh blood was centrifuged at $2000 \mathrm{~g}$ for $10 \mathrm{~min}$, and then, the supernatant was followed by a second centrifugation at $5000 \mathrm{~g}$ for $15 \mathrm{~min}$ at $4^{\circ} \mathrm{C}$. In addition, primary OSCC and paired adjacent noncancerous tissues were also obtained from 47 out of 184 patients. All samples were stored at $-80^{\circ} \mathrm{C}$ until analysis. Written informed consent was obtained from all participants. Clinicopathological information including age, gender, and TNM stage was collected by reviewing the medical records. All patients were followed up for 3 years to record survival conditions. The median follow-up period was 31 months (range, 5-36 months).

2.2. Isolation of Exosomes from Plasma. Exosomes were extracted from plasma using the ExoQuick ULTRA EV Isolation Kit for Serum and Plasma (SBI System Biosciences, US) according to the manufacturer's protocol. Briefly, ExoQuick ULTRA was added to $250 \mu \mathrm{L}$ plasma and incubated on ice for $30 \mathrm{~min}$; after that, the mixture was centrifuged at $3000 \mathrm{~g}$ for $10 \mathrm{~min}$. Extracellular vesicles (EVs) in the pellet were resuspended and added to prewashed ExoQuick ULTRA columns, and then, the purified exosomes were obtained after a second centrifugation at $1000 \mathrm{~g}$ for $30 \mathrm{~s}$.

2.3. Quantification of Exosomal miRNAs. Total RNA of exosomes was extracted using the miRNeasy Serum/Plasma Kit (Qiagen, Germany) according to the manufacturer instructions, while total RNA of tumor tissues and paired adjacent noncancerous tissues were extracted using the miRNeasy Mini Kit (Qiagen, Germany). RNA quality was assessed using a NanoDrop ${ }^{\mathrm{rm}} 8000$ spectrophotometer (Thermo Scientific, US). Complementary DNA (cDNA) was synthesized using TaqMan MicroRNA primers specific for miR-130a and a TaqMan MicroRNA Reverse-Transcription Kit (Thermo Scientific, US). Quantitative reverse-transcription polymerase chain reaction (qRT-PCR) was performed in triplicate on the Bio-Rad CFX Connect Real-Time PCR Detection System (Bio-rad, US). The PCR conditions were $95^{\circ} \mathrm{C}$ for $10 \mathrm{~min}$, followed by 40 cycles of $95^{\circ} \mathrm{C}$ for $15 \mathrm{~s}$ and $60^{\circ} \mathrm{C}$ for $1 \mathrm{~min}$. In addition, a synthetic Caenorhabditis elegans miR-39 RNA oligonucleotide (cel-miR-39 mimic) was used as a spike-in control in plasma exosomes, while U6 small nuclear RNA was used as an internal control in tissues. Relative quantification of miR-130a expression was calculated using the $2^{-\Delta \Delta \mathrm{CT}}$ method.

2.4. Statistical Analysis. Statistical analyses were performed using the SPSS statistical software package (version 19.0, SPSS lnc., US) and GraphPad Prism (version 5.0, GraphPad Software Inc., US). Student's $t$-test was used to determine statistically significant differences between groups. The association between miR-130a expression and clinicopathological characteristics was analyzed by the $\chi^{2}$ test. Receiver operating characteristic (ROC) curves were established to evaluate the diagnostic value of miR-130a, and the area under the curve (AUC) with 95\% confidence interval (CI) was also calculated. Overall survival (OS) and recurrencefree survival (RFS) curves were constructed using the Kaplan-Meier method, and the differences were examined using log-rank tests. Univariate and multivariate hazard ratios for OS and RFS were estimated using a Cox proportional hazards regression model. A two-tailed $p$ value $<0.05$ was considered statistically significant. 


\section{Results}

3.1. Characteristics of the Study Population. The clinical characteristics of the 184 OSCC patients and 196 healthy volunteers are summarized in Table 1 . The control individuals were frequently matched with patients by age and gender. The mean ages of the patients and the controls were $56.3 \pm 16.4$ and $55.5 \pm 16.6$ years, respectively $(p=0.947)$. No significantly statistical differences were noted in the distributions of the smoking habit $(p=0.12)$ and drinking habit ( $p=0.475$ ) between the patients and controls. Most of the patients had early $\mathrm{T}$-stage, negative $\mathrm{N}$-stage, and welldifferentiated or moderately differentiated OSCC. Eighty patients were diagnosed at advanced TNM stage. Ninety patients had experienced tumor recurrence after surgical resection, and 63 patients had died at the end of 3-year follow-up. The median RFS was 28.9 months (Supplementary File 1).

\subsection{Expression of miR-130a in OSCC Tissues and Plasma} Exosomes. Expression of miR-130a was assessed by qRTPCR. As shown in Figure 1(a), the results showed that the relative expression levels of exosomal miR-130a were significantly higher in OSCC patients than those of the healthy controls $(p<0.0001)$. For OSCC patients, the expression of miR-130a was significantly upregulated in OSCC tissues $(54.5 \pm 12.6$, mean relative expression level \pm SD) when compared with that in paired adjacent noncancerous tissues $(41.5 \pm 11.6$, Figure 1(b)). Furthermore, the correlation between exosomal miR-130a levels and miR-130a expression in OSCC tissues in the same patients were examined, and a significant positive correlation was demonstrated between them $(p<0.001$, Figure $1(\mathrm{~d}))$. We also compared the expression levels of exosomal miR-130a in OSCC patients with different TNM stages, and miR-130a levels were still significantly lower in healthy controls than OSCC patients with TNM stage I ( $p<0.001)$, as well as TNM stage II, III, and IV (Figure 1(e) and Table 2). In addition, the results also showed that miR-130a levels were significantly higher in stage III and IV compared to stage I and II ( $p=0.009$, Table 2$)$.

3.3. Exosomal miR-130a as Diagnosis Signature for OSCC. ROC curve analysis was performed to know whether exosomal miR-130a could sensitively and specifically discriminate the OSCC patients from the healthy controls. The area under the curve (AUC) value of exosomal miR-130a was $0.812(95 \%$ confidence interval $(\mathrm{CI})=0.77-0.853$, Figure 1(c)). An optimal cut-off value was indicated at 15.85 with a sensitivity of $98.5 \%$ and a specificity of $45.7 \%$. Furthermore, the AUC value obtained for exosomal miR-130a to distinguish the OSCC patients in the early stage (I and II) from the healthy controls was $0.769(95 \% \mathrm{CI}=0.715-0.823$, Figure 1(f)), and an optimal cut-off value was indicated at 10.05 with a sensitivity of $100 \%$ and a specificity of $38.1 \%$. These results indicated that exosomal miR-130a might have a potential diagnostic value for distinguishing the OSCC patients from the healthy controls.
TABLE 1: Clinical characteristics of participants.

\begin{tabular}{|c|c|c|c|}
\hline Variable & $\begin{array}{l}\text { Patient } \\
(N=184)\end{array}$ & $\begin{array}{l}\text { Control } \\
(N=196)\end{array}$ & $\begin{array}{c}p \\
\text { value }\end{array}$ \\
\hline $\begin{array}{l}\text { Age (years, } \\
\text { mean } \pm S D)\end{array}$ & $56.3 \pm 16.4$ & $55.5 \pm 16.6$ & 0.947 \\
\hline \multicolumn{4}{|c|}{ Age group (years), N (\%) } \\
\hline $\begin{array}{l}\leq 60 \\
>60\end{array}$ & $\begin{array}{c}82(44.6) \\
102(55.4)\end{array}$ & $\begin{array}{c}87(44.4) \\
109(55.6)\end{array}$ & 0.972 \\
\hline \multicolumn{4}{|l|}{ Gender, N (\%) } \\
\hline $\begin{array}{l}\text { Male } \\
\text { Female }\end{array}$ & $\begin{array}{l}125(67.9) \\
59(32.1)\end{array}$ & $\begin{array}{l}134(68.4) \\
62(31.6)\end{array}$ & 0.928 \\
\hline \multicolumn{4}{|c|}{ Smoking habit, N (\%) } \\
\hline $\begin{array}{l}\text { Yes } \\
\text { No } \\
\end{array}$ & $\begin{array}{c}102(55.4) \\
82(44.6) \\
\end{array}$ & $\begin{array}{c}93(47.4) \\
103(52.6) \\
\end{array}$ & 0.12 \\
\hline \multicolumn{4}{|c|}{ Drinking habit, N (\%) } \\
\hline $\begin{array}{l}\text { Yes } \\
\text { No }\end{array}$ & $\begin{array}{c}134(72.8) \\
50(27.2)\end{array}$ & $\begin{array}{c}149(76) \\
47(24)\end{array}$ & 0.475 \\
\hline \multicolumn{4}{|l|}{ Tumor site, $N(\%)$} \\
\hline $\begin{array}{l}\text { Tongue } \\
\text { Nontongue }\end{array}$ & $\begin{array}{c}83(45.1) \\
101(54.9)\end{array}$ & & \\
\hline \multicolumn{4}{|l|}{ T-stage, N (\%) } \\
\hline $\begin{array}{l}\mathrm{T} 1+\mathrm{T} 2 \\
\mathrm{~T} 3+\mathrm{T} 4\end{array}$ & $\begin{array}{l}111(60.3) \\
73(39.7)\end{array}$ & & \\
\hline \multicolumn{4}{|l|}{ N-stage, $N(\%)$} \\
\hline $\begin{array}{l}\text { Positive } \\
\text { Negative }\end{array}$ & $\begin{array}{c}69(37.5) \\
115(62.5)\end{array}$ & & \\
\hline \multicolumn{4}{|l|}{ TNM stage, $N(\%)$} \\
\hline $\begin{array}{l}\text { I-II } \\
\text { III-IV }\end{array}$ & $\begin{array}{l}104(56.5) \\
80(43.5)\end{array}$ & & \\
\hline \multicolumn{4}{|c|}{ Histologic grade, $N(\%)$} \\
\hline $\begin{array}{l}\text { Well + moderate } \\
\text { Poor }\end{array}$ & $\begin{array}{l}120(65.2) \\
64(34.8)\end{array}$ & & \\
\hline
\end{tabular}

${ }^{*}$ Mean $p$ value $<0.05$.

3.4. Clinicopathological Significance of Exosomal miR-130a Expression. For clinicopathological analysis, patients were classified into high- and low-expression groups using the median relative expression level of exosomal miR-130a. Our results revealed that high expression of exosomal miR-130a was significantly correlated with the late T-stage $(p=0.024)$, advanced TNM-stage $(p=0.003)$, and poorly differentiated OSCC $(p=0.013)$. However, there were no significant correlations of exosomal miR-130a expression with other clinical variables such as the age, gender, smoking and drinking habit, tumor site, and $\mathrm{N}$-stage (Table 3).

\subsection{Correlation between Exosomal miR-130a Expression and} Prognosis of OSCC Patients. To evaluate the prognostic significance of exosomal miR-130a expression, the Kaplan-Meier OS and RFS curves of the 184 OSCC patients were compared between those with high and those with low levels of exosomal miR-130a. The results showed that OSCC patients with high exosomal miR-130a expression had shorter 3-year OS $(p=0.0018$, Figure 2(a)) and RFS $(p=0.0005$, Figure 2(b)) than those with low exosomal miR130a expression. Univariate analysis showed that exosomal miR-130a expression levels, T-stage, TNM stage, and histologic grade were significantly correlated with OS and RFS 


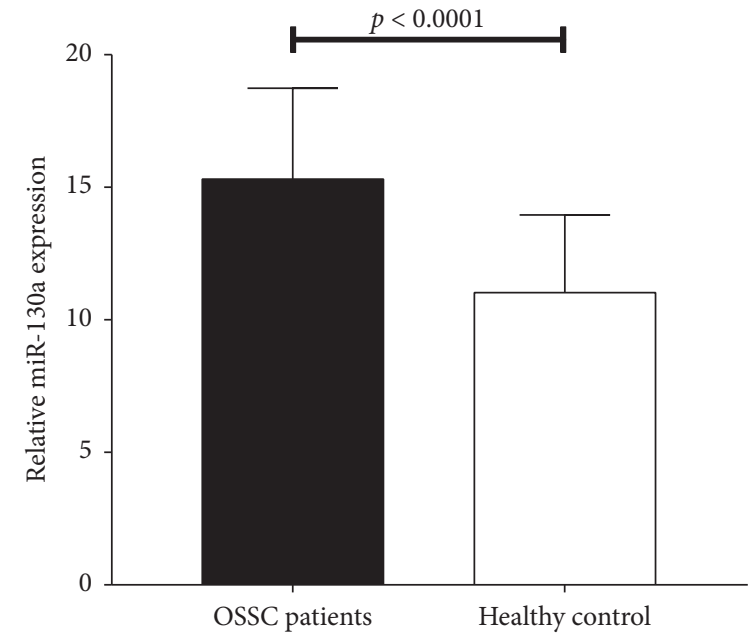

(a)

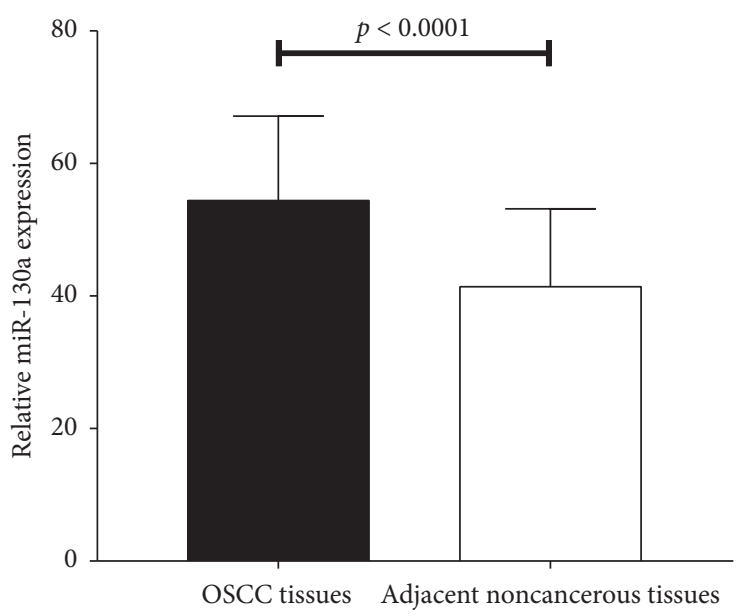

(b)

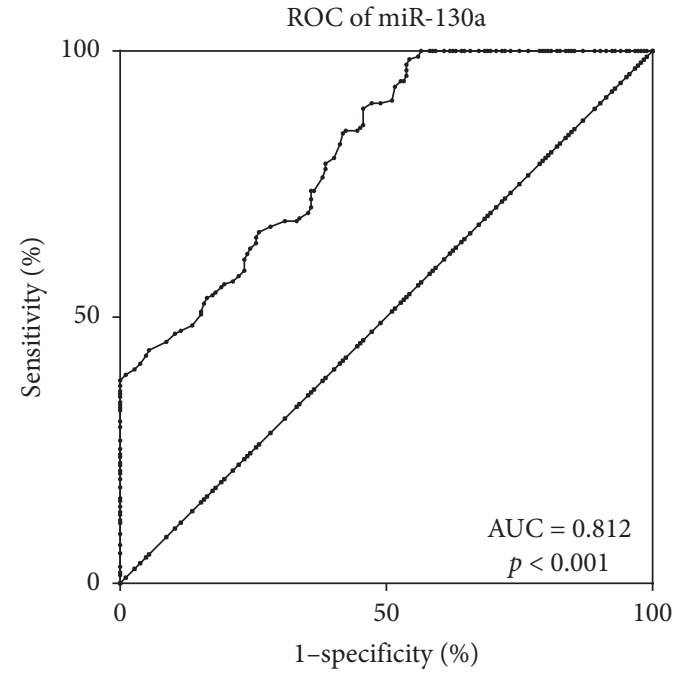

(c)

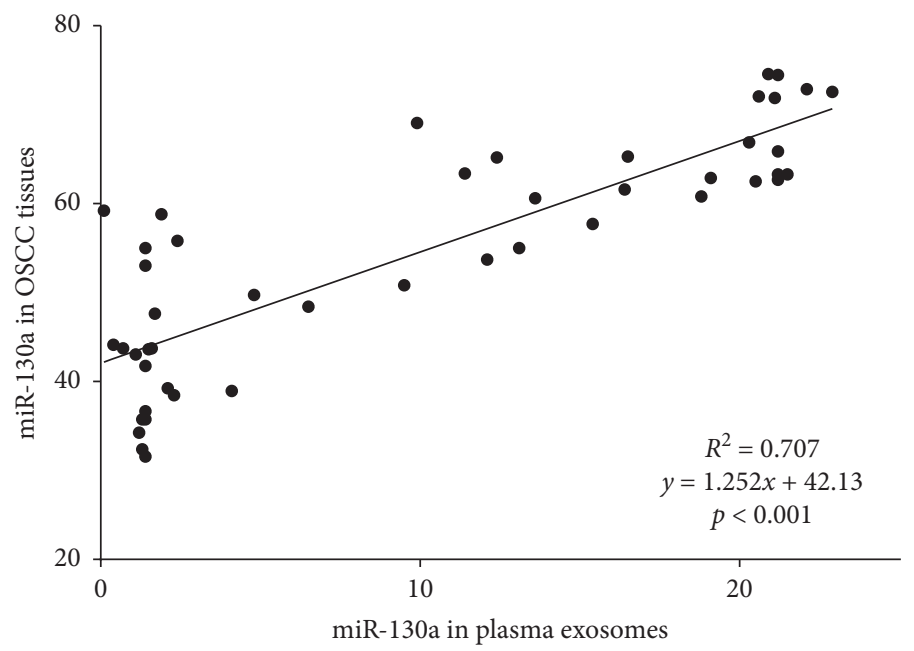

(d)

FIgURE 1: Continued. 


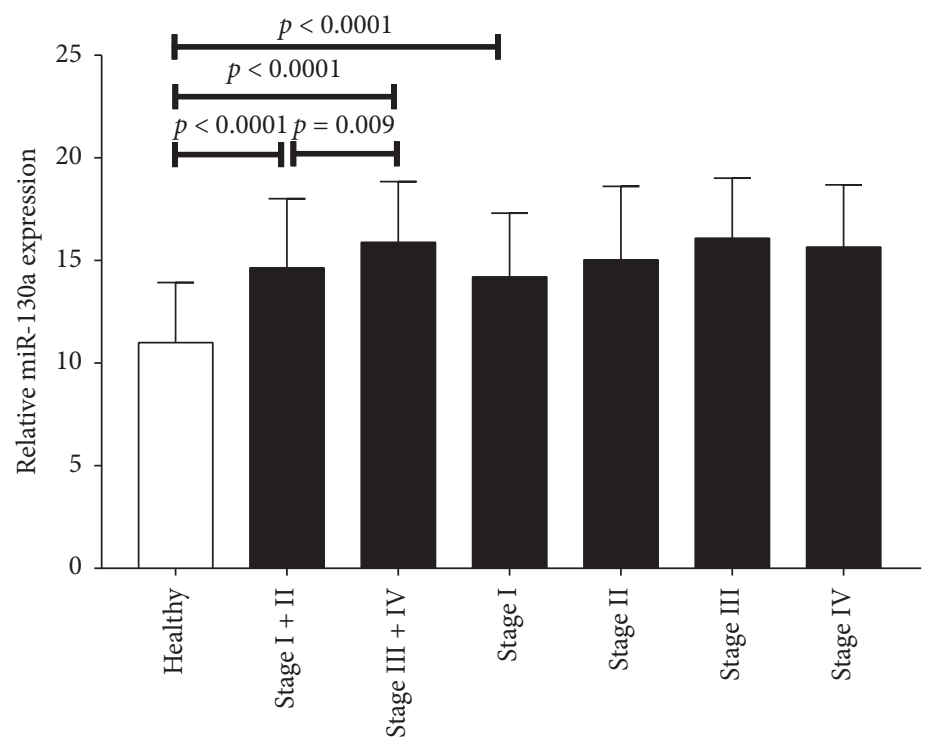

(e)

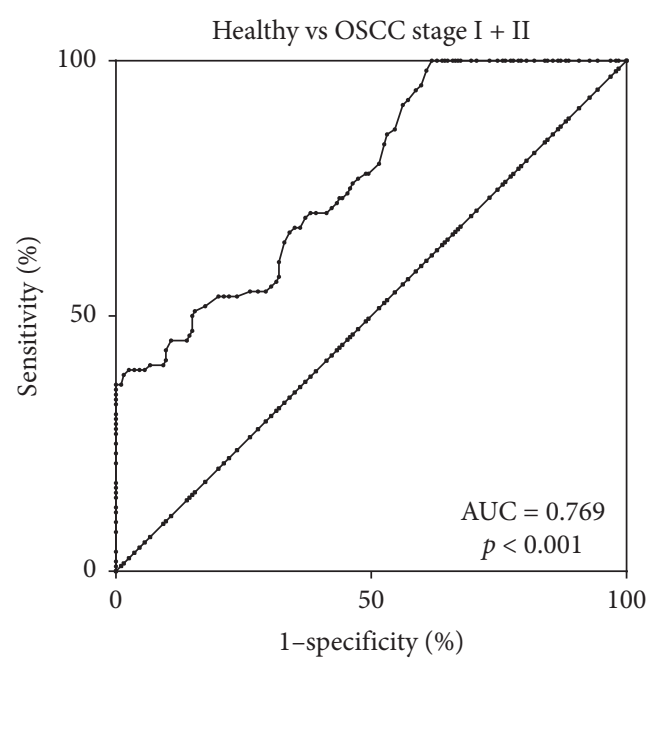

(f)

FIGURE 1: miR-130a expression is significantly higher in OSCC patients. (a) Comparison of exosomal miR-130a expression levels between OSCC patients and healthy controls. (b) Comparison of miR-130a expression levels between OSCC tissues and paired adjacent noncancerous tissues. (c) ROC curves of exosomal miR-130a for discriminating OSCC patients from healthy controls. (d) Correlation between expression of miR-130a in plasma exosomes and that in OSCC tissues. (e) Comparison of miR-130a expression levels between OSCC patients at different TNM stages and healthy controls. (f) ROC curves of exosomal miR-130a for discriminating patients with early-stage OSCC from healthy controls.

TABLE 2: Performance of exosomal miR-130a for detection of OSCC with different TNM stages.

\begin{tabular}{lccccccc}
\hline Group & Healthy control & Stage I + II & Stage III + IV & Stage I & Stage II & Stage III & Stage IV \\
\hline Healthy control & - & - & - & - & - & - & - \\
Stage I + II & $<0.0001^{*}$ & - & - & - & - & - & - \\
Stage III + IV & $<0.0001^{*}$ & $0.009^{*}$ & - & - & - & - & - \\
Stage I & $<0.0001^{*}$ & 0.5 & $0.002^{*}$ & - & - & - & - \\
Stage II & $<0.0001^{*}$ & 0.533 & 0.164 & 0.262 & - & - & - \\
Stage III & $<0.0001^{*}$ & $0.015^{*}$ & 0.706 & $0.005^{*}$ & 0.133 & - & - \\
Stage IV & $<0.0001^{*}$ & 0.084 & 0.687 & $0.02^{*}$ & 0.417 & 0.501 & - \\
\hline
\end{tabular}

${ }^{*}$ Mean $p$ value $<0.05$.

$(p<0.05)$, whereas age, gender, smoking and drinking habit, tumor site, or $\mathrm{N}$-stage were not (Tables 4 and 5). Multivariate Cox regression analysis was performed for those factors which showed significance in univariate analysis. Also, our results showed that exosomal miR-130a expression levels $(p=0.001)$, TNM stage $(p=0.037)$, and histologic grade ( $p=0.025)$ were independent prognostic factors for predicting the 3-year OS of OSCC patients (Table 4). Furthermore, in RFS, multivariate analysis demonstrated that exosomal miR-130a expression levels $(p=0.003)$, T-stage $(p=0.028)$, TNM stage $(p=0.03)$, and histologic grade ( $p=0.043)$ emerged as significant independent prognostic factors (Table 5).

\section{Discussion}

In recent years, liquid biopsy has received much more attention as a novel noninvasive diagnostic or prognostic tool for providing real-time information about cancer, which was based on the detection of circulating tumour DNA (ctDNA), circulating tumour cells (CTCs), exosomal miRNAs, and so on [28]. Analyses of these CTCs or circulating nucleic acids could be applied to cancer early diagnosis, response monitoring, and drug resistance assessment [28]. The key advantages of liquid biopsy are that it could provide greater accuracy of tumour heterogeneity than tissue-based biopsy and collect samples in a minimally invasive way. Particularly, exosomal miRNAs have been proved to be existed not only in blood but also in other multiple body fluids, such as urine, saliva, pleural effusions, and cerebrospinal fluid [9]. Up to now, at least 50 exosomal miRNAs have been proved to play a role in cancer diagnosis and prognosis $[29,30]$. However, to the best of our knowledge, there is only one study by $\mathrm{He}$ et al. which demonstrated that salivary exosomal miR-24-3p could be used as a candidate screening biomarker for OSCC and it can enhance the proliferative capacity of OSCC cells through targeting PER 1 [21]. In the present study, we provided the first investigation in the diagnostic and 
TABLE 3: Clinicopathological characteristics of patients with OSCC.

\begin{tabular}{|c|c|c|c|c|}
\hline \multirow{2}{*}{ Clinicopathological factors } & \multirow{2}{*}{ No. } & \multicolumn{3}{|c|}{ Exosomal miR-130a expression } \\
\hline & & Low, N (\%) & High, N (\%) & $p$ value \\
\hline \multicolumn{5}{|l|}{ Age } \\
\hline$<60$ years & 82 & $43(23.4)$ & $39(21.2)$ & \multirow{2}{*}{0.553} \\
\hline$\geq 60$ years & 102 & $49(26.6)$ & $53(28.8)$ & \\
\hline \multicolumn{5}{|l|}{ Gender } \\
\hline Male & 125 & $59(32.1)$ & $66(35.9)$ & \multirow{2}{*}{0.269} \\
\hline Female & 59 & $33(17.9)$ & $26(14.1)$ & \\
\hline \multicolumn{5}{|l|}{ Smoking habit } \\
\hline Yes & 102 & $50(27.2)$ & $52(28.3)$ & \multirow{2}{*}{0.767} \\
\hline No & 82 & $42(22.8)$ & $40(21.7)$ & \\
\hline \multicolumn{5}{|l|}{ Drinking habit } \\
\hline Yes & 134 & $65(35.3)$ & $69(37.5)$ & \multirow{2}{*}{0.507} \\
\hline No & 50 & $27(14.7)$ & $23(12.5)$ & \\
\hline \multicolumn{5}{|l|}{ Tumor site } \\
\hline Tongue & 83 & $43(23.4)$ & $40(21.7)$ & \multirow{2}{*}{0.657} \\
\hline Nontongue & 101 & $49(26.6)$ & $52(28.3)$ & \\
\hline \multicolumn{5}{|l|}{ T-stage } \\
\hline $\mathrm{T} 1+\mathrm{T} 2$ & 111 & $63(34.2)$ & $48(26.1)$ & \multirow{2}{*}{$0.024^{*}$} \\
\hline $\mathrm{T} 3+\mathrm{T} 4$ & 73 & $29(15.8)$ & $44(23.9)$ & \\
\hline \multicolumn{5}{|l|}{$N$-stage } \\
\hline Negative & 115 & $63(34.2)$ & $52(28.3)$ & \multirow{2}{*}{0.094} \\
\hline Positive & 69 & $29(15.8)$ & $40(21.7)$ & \\
\hline \multicolumn{5}{|l|}{ TNM stage } \\
\hline I-II & 104 & $62(33.7)$ & $42(22.8)$ & \multirow{2}{*}{$0.003^{*}$} \\
\hline III-IV & 80 & $30(16.3)$ & $50(27.2)$ & \\
\hline \multicolumn{5}{|l|}{ Histologic grade } \\
\hline Well + moderate & 120 & $68(37)$ & $52(28.3)$ & \multirow[b]{2}{*}{$0.013^{*}$} \\
\hline Poor & 64 & $24(13)$ & $40(21.7)$ & \\
\hline
\end{tabular}

${ }^{*}$ Mean $p$ value $<0.05$.

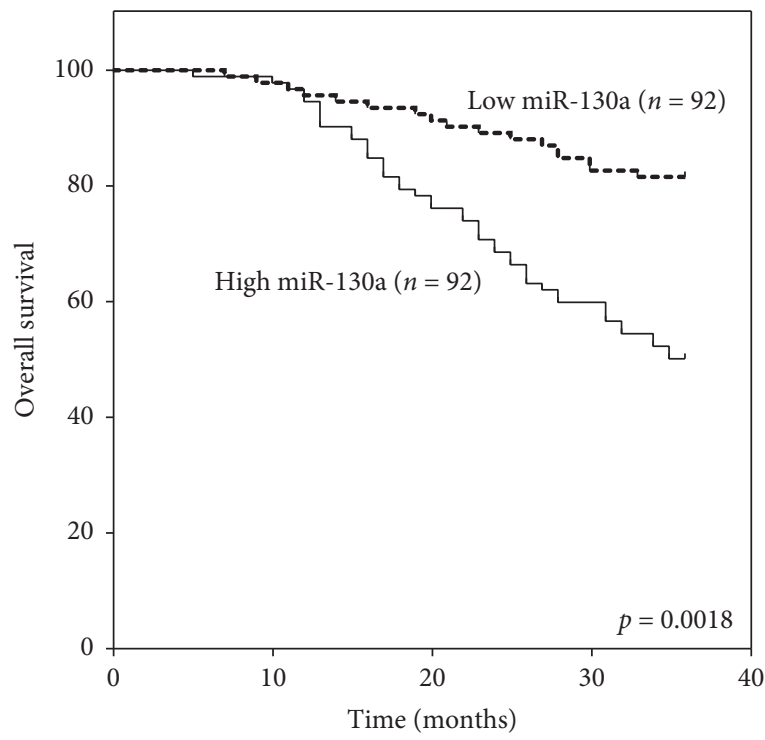

(a)

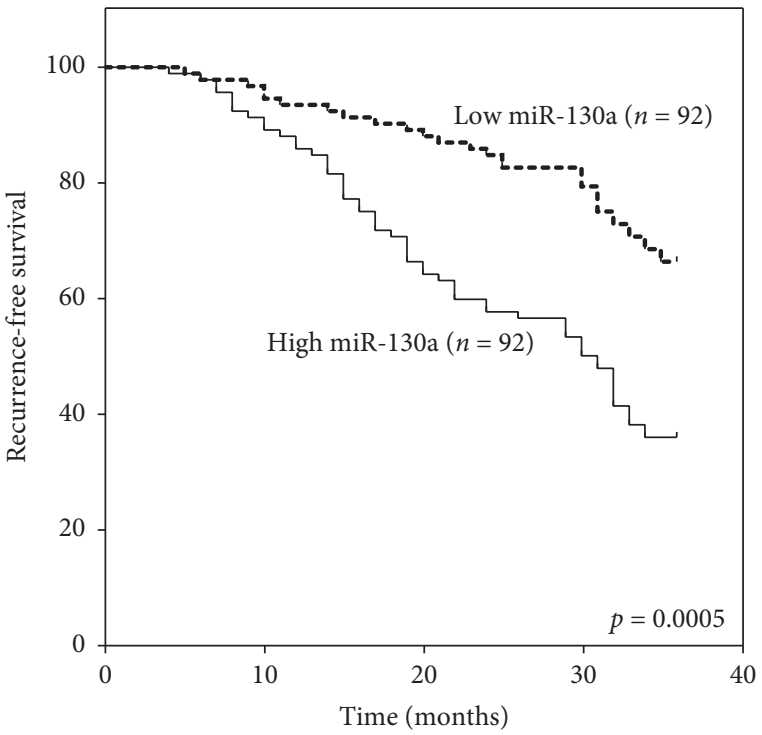

(b)

FIGURE 2: Survival analysis of 184 patients with OSCC by the Kaplan-Meier method. Overall survival and recurrence-free survival of patients with high and low expression of plasma-derived exosomal miR-130a. $p$ values were calculated using a two-sided log-rank test. 
TABLE 4: Univariate and multivariate analyses of overall survival in 184 patients with OSCC.

\begin{tabular}{|c|c|c|c|c|}
\hline \multirow{2}{*}{ Variable } & \multicolumn{2}{|c|}{ Univariate analysis } & \multicolumn{2}{|c|}{ Multivariate analysis } \\
\hline & HR (95\% CI) & $p$ value & HR (95\% CI) & $p$ value \\
\hline Exosomal miR-130a (high) & $4.412(2.265-8.591)$ & $<0.001^{*}$ & $3.276(1.624-6.607)$ & $0.001^{*}$ \\
\hline Age ( $\geq 60$ years $)$ & $1.007(0.546-1.859)$ & 0.981 & & \\
\hline Gender (male) & $1.358(0.713-2.585)$ & 0.352 & & \\
\hline Smoking habit (yes) & $1.206(0.654-2.224)$ & 0.548 & & \\
\hline Drinking habit (yes) & $1.482(0.728-3.016)$ & 0.277 & & \\
\hline Tumor site (tongue) & $1.039(0.555-1.948)$ & 0.904 & & \\
\hline T-stage (T3 + T4) & $2.227(1.195-4.153)$ & $0.012^{*}$ & $1.853(0.933-3.682)$ & 0.078 \\
\hline N-stage (positive) & $1.546(0.831-2.877)$ & 0.169 & & \\
\hline TNM stage (III-IV) & $2.581(1.382-4.821)$ & $0.003^{*}$ & $2.07(1.046-4.097)$ & $0.037^{*}$ \\
\hline Histologic grade (poor) & $2.871(1.517-5.434)$ & $0.001^{*}$ & $2.199(1.102-4.389)$ & $0.025^{*}$ \\
\hline
\end{tabular}

HR: hazard ratio; CI: confidence interval; * mean $p$ value $<0.05$.

TABLe 5: Univariate and multivariate analyses of recurrence-free survival in 184 patients with OSCC.

\begin{tabular}{lccc}
\hline Variable & Univariate analysis & & Multivariate analysis \\
& HR $(95 \% \mathrm{CI})$ & $p$ value & HR (95\% CI) \\
\hline Exosomal miR-130a (high) & $3.518(1.917-6.455)$ & $<0.001^{*}$ & $2.627(1.382-4.993)$ \\
Age ( $\geq 60$ years) & $1.181(0.66-2.114)$ & 0.575 & $0.003^{*}$ \\
Gender (male) & $1.121(0.603-2.082)$ & 0.718 & \\
Smoking habit (yes) & $1.41(0.786-2.527)$ & 0.88 & \\
Drinking habit (yes) & $1.051(0.549-2.014)$ & 0.819 & $2.086(1.082-4.023)$ \\
Tumor site (tongue) & $1.072(0.59-1.948)$ & $0.005^{*}$ & $0.028^{*}$ \\
T-stage (T3+ T4) & $2.357(1.287-4.317)$ & 0.098 & \\
N-stage (positive) & $1.64(0.913-2.946)$ & $0.004^{*}$ & $2.056(1.073-3.936)$ \\
TNM stage (III-IV) & $2.429(1.336-4.414)$ & $0.003^{*}$ & $2.007(1.023-3.938)$ \\
Histologic grade (poor) & $2.583(1.38-4.836)$ & $0.033^{*}$ \\
\hline
\end{tabular}

HR: hazard ratio; CI: confidence interval; ${ }^{*}$ mean $p$ value $<0.05$.

prognostic value of plasma-derived exosomal miR-130a expression in OSCC.

The previous study by Hilly et al. provided the first evidence that miR-130a expression in OSCC tissues from young patients with recurrence was upregulated as compared with the patients without recurrence [31]. In a recent study by Yang et al., it was also reported that miR-130a was highly expressed in OSCC tissues and significantly associated with the TNM stage and lymph node metastasis [32]. Consistent with that, in our study, we found that the expression of miR-130a was upregulated in OSCC tissues when compared with that in paired adjacent noncancerous tissues, and it was significantly associated with more aggressive clinicopathological characteristics of OSCC patients, including late T-stage, advanced TNM stage, and poorly differentiated grade. Previous studies have reported that miR-130a could facilitate proliferation and metastasis of tumor cells through downregulating the expression of tumor suppressor genes, such as phosphatase and tensin homolog (PTEN) in osteosarcoma and breast cancer [25, 33], peroxisome proliferator-activated receptor gamma (PPARG) in chorangiocarcinoma [34], and collapsing-response mediator protein type 4 (CRMP4) in gastric cancer [35]. In two oral tongue squamous cell carcinoma (OTSCC) cell lines CAL-27 and SSC-4, cylindromatosis (CYLD), which acts as a tumor suppressor in several malignancies and is responsible for cisplatin resistance in OSCC patients $[36,37]$, has been proved to be one of the multiple target genes of miR-130a [32]. These are supposed to be the mechanisms of its correlation with more aggressive tumors. Further studies are needed. Moreover, our results also demonstrated that the expression of plasma-derived exosomal miR-130a was significantly higher in OSCC patients than that of the healthy volunteers. Also, there was a significant positive correlation between expression of miR-130a in plasma exosomes and primary OSCC tissues, suggesting that tumor tissue may be the source of circulating exosomal miRNAs. ROC curve analysis revealed that exosomal miR-130a holds great promise to be used as a novel diagnostic biomarker for the screening of OSCC.

Accumulating evidence has suggested that miR-130a could be used as potential prognostic biomarkers for certain types of cancer. Fox example, a study by Jiang $\mathrm{H}$ et al. reported that high expression of miR-130a promoted gastric cancer tumorigenesis by targeting runt-related transcription factor 3 (RUNX3) and was significantly associated with poor OS [38]. Contrastingly, Kaplan-Meier analysis by Li B et al. demonstrated that high expression of miR-130a in patients with hepatocellular carcinoma (HCC) had significantly improved OS [23]. In a recent meta-analysis, Peng et al. evaluated 12 eligible studies comprising 1582 cases with various types of cancer, including HCC, colorectal cancer, gastric cancer, lymphoma, non-small-cell lung cancer, cervical cancer, cholangiocarcinoma, and osteosarcoma, and the pooled 
hazard ratios (HRs) indicated a significant relationship between the high levels of miR-130a expression and poor OS in cancer patients $(\mathrm{HR}=1.58,95 \% \mathrm{CI}=1.21-2.06, p<0.001)$ [39]. Up to now, the prognostic role of miR-130a has not been investigated in OSCC. In the present study, our results showed that OSCC patients with high exosomal miR-130a expression had a significantly worse 3-year OS and RFS than those with low exosomal miR-130a expression. Also, multivariate analysis further confirmed that miR-130a was a prognostic biomarker independent of several adjusted well-known prognostic parameters for OSCC, including T-stage, TNM stage, and histologic grade. However, the small sample size, the retrospective nature of this study, and the lack of underlying biological mechanisms for the effects of miR-130a on the occurrence, development, and prognosis of OSCC did not allow us to draw any concrete conclusions yet. Further studies are required to confirm the present findings. Moreover, it is probably meaningful to monitor the changes of exosomal miR-130a levels after surgery because it might be associated with tumor recurrence, side effects of treatment, survival quality, and so on, and further studies are also required.

\section{Conclusions}

In conclusion, our results provided the first evidence that plasma-derived exosomal miR-130a expression was significantly increased in OSCC patients and associated with late T-stage, advanced TNM stage, and poorly differentiated grade. Moreover, exosomal miR-130a may serve as a promising diagnostic and prognostic biomarker for OSCC patients.

\section{Data Availability}

The data used to support the findings of this study are available from the corresponding author upon request.

\section{Conflicts of Interest}

The authors declare that there are no conflicts of interest.

\section{Authors' Contributions}

$\mathrm{TH}$ contributed to the conception, design, sample processing, statistical analysis, and interpretation of data. XYG and XL contributed to the collection of human samples and clinical and demographic data. CJL and XRW contributed to the sample processing, data analysis, and interpretation of data. $\mathrm{KH}$ contributed to the conception, interpretation of data, and the draft and critical revision of the manuscript.

\section{Acknowledgments}

The authors would like to thank all the participants who took part in this study.

\section{Supplementary Materials}

Table 1: clinical characteristics of patients. Table 2: clinical characteristics of controls. (Supplementary Materials)

\section{References}

[1] F. Bray, J. Ferlay, I. Soerjomataram, R. L. Siegel, L. A. Torre, and A. Jemal, "Global cancer statistics 2018: GLOBOCAN estimates of incidence and mortality worldwide for 36 cancers in 185 countries," CA: A Cancer Journal for Clinicians, vol. 68, no. 6, pp. 394-424, 2018.

[2] W. Chen, R. Zheng, P. D. Baade et al., "Cancer statistics in China, 2015," CA: A Cancer Journal for Clinicians, vol. 66, no. 2, pp. 115-132, 2016.

[3] C. Rivera, "Essentials of oral cancer," International Journal of Clinical and Experimental Pathology, vol. 8, no. 9, pp. 11884-11894, 2015.

[4] P. H. Montero and S. G. Patel, "Cancer of the oral cavity," Surgical Oncology Clinics of North America, vol. 24, no. 3, pp. 491-508, 2015.

[5] M. Mascitti, G. Orsini, and V. Tosco, "An overview on current non-invasive diagnostic devices in oral oncology," Frontiers in Physiology, vol. 9, p. 1510, 2018.

[6] E. R. Abels and X. O. Breakefield, "Introduction to extracellular vesicles: biogenesis, rna cargo selection, content, release, and uptake," Cellular and Molecular Neurobiology, vol. 36, no. 3, pp. 301-312, 2016.

[7] M. Record, K. Carayon, M. Poirot, and S. Silvente-Poirot, "Exosomes as new vesicular lipid transporters involved in cellcell communication and various pathophysiologies," Biochimica et Biophysica Acta (BBA)-Molecular and Cell Biology of Lipids, vol. 1841, no. 1, pp. 108-120, 2014.

[8] A. Srivastava, J. Filant, K. Moxley, A. Sood, S. McMeekin, and R. Ramesh, "Exosomes: a role for naturally occurring nanovesicles in cancer growth, diagnosis and treatment," Current Gene Therapy, vol. 15, no. 2, pp. 182-192, 2015.

[9] J. C. Akers, D. Gonda, R. Kim, B. S. Carter, and C. C. Chen, "Biogenesis of extracellular vesicles (EV): exosomes, microvesicles, retrovirus-like vesicles, and apoptotic bodies," Journal of Neuro-Oncology, vol. 113, no. 1, pp. 1-11, 2013.

[10] J. Ratajczak, M. Wysoczynski, F. Hayek, A. JanowskaWieczorek, and M. Z. Ratajczak, "Membrane-derived microvesicles: important and underappreciated mediators of cell-to-cell communication," Leukemia, vol. 20, no. 9, pp. 1487-1495, 2006.

[11] H. Peinado, S. Lavotshkin, and D. Lyden, "The secreted factors responsible for pre-metastatic niche formation: old sayings and new thoughts," Seminars in Cancer Biology, vol. 21, no. 2, pp. 139-146, 2011.

[12] A. Izzotti, S. Carozzo, A. Pulliero, D. Zhabayeva, J. L. Ravetti, and R. Bersimbaev, "Extracellular MicroRNA in liquid biopsy: applicability in cancer diagnosis and prevention," American Journal of Cancer Research, vol. 6, no. 7, pp. 1461-1493, 2016.

[13] D. H. Chitwood and M. C. P. Timmermans, "Small RNAs are on the move," Nature, vol. 467, no. 7314, pp. 415-419, 2010.

[14] M. Acunzo, G. Romano, D. Wernicke, and C. M. Croce, "MicroRNA and cancer - a brief overview," Advances in Biological Regulation, vol. 57, pp. 1-9, 2015.

[15] M. Tkach and C. Théry, "Communication by extracellular vesicles: where we are and where we need to go," Cell, vol. 164, no. 6, pp. 1226-1232, 2016.

[16] Q. Ge, Y. Zhou, J. Lu, Y. Bai, X. Xie, and Z. Lu, "miRNA in plasma exosome is stable under different storage conditions," Molecules, vol. 19, no. 2, pp. 1568-1575, 2014.

[17] Q. Liu, Z. Yu, S. Yuan et al., "Circulating exosomal microRNAs as prognostic biomarkers for non-small-cell lung cancer," Oncotarget, vol. 8, no. 8, pp. 13048-13058, 2017. 
[18] X. Xue, Y. Zhao, X. Wang, L. Qin, and R. Hu, "Development and validation of serum exosomal microRNAs as diagnostic and prognostic biomarkers for hepatocellular carcinoma," Journal of Cellular Biochemistry, vol. 120, no. 1, pp. 135-142, 2019.

[19] S. Yan, Y. Jiang, C. Liang et al., "Exosomal miR-6803-5p as potential diagnostic and prognostic marker in colorectal cancer," Journal of Cellular Biochemistry, vol. 119, no. 5, pp. 4113-4119, 2018.

[20] M. Wang, S. Ji, G. Shao et al., "Effect of exosome biomarkers for diagnosis and prognosis of breast cancer patients," Clinical and Translational Oncology, vol. 20, no. 7, pp. 906-911, 2018.

[21] L. He, F. Ping, and Z. Fan, "Salivary exosomal miR-24-3p serves as a potential detective biomarker for oral squamous cell carcinoma screening," Biomedicine \& Pharmacotherapy, vol. 121, Article ID 109553, 2020.

[22] L. P. Sun, K. Xu, and J. Cui, "Cancer-associated fibroblastderived exosomal miR-382-5p promotes the migration and invasion of oral squamous cell carcinoma," Oncology Reports, vol. 42, no. 4, pp. 1319-1328, 2019.

[23] B. Li, P. Huang, J. Qiu, Y. Liao, J. Hong, and Y. Yuan, "MicroRNA-130a is down-regulated in hepatocellular carcinoma and associates with poor prognosis," Medical Oncology, vol. 31, no. 10, p. 230, 2014.

[24] K. Boll, K. Reiche, K. Kasack et al., "MiR-130a, miR-203 and miR-205 jointly repress key oncogenic pathways and are downregulated in prostate carcinoma," Oncogene, vol. 32, no. 3, pp. 277-285, 2013.

[25] H. Wei, R. Cui, J. Bahr et al., "miR-130a deregulates pten and stimulates tumor growth," Cancer Research, vol. 77, no. 22, pp. 6168-6178, 2017.

[26] X. Zhang, L. Huang, Y. Zhao, and W. Tan, "Downregulation of miR-130a contributes to cisplatin resistance in ovarian cancer cells by targeting X-linked inhibitor of apoptosis (XIAP) directly," Acta Biochimica et Biophysica Sinica, vol. 45, no. 12, pp. 995-1001, 2013.

[27] L. He, H.-Y. Wang, L. Zhang et al., "Prognostic significance of low DICER expression regulated by miR-130a in cervical cancer," Cell Death \& Disease, vol. 5, no. 5, p. e1205, 2014.

[28] G. Siravegna, S. Marsoni, S. Siena, and A. Bardelli, "Integrating liquid biopsies into the management of cancer," Nature Reviews Clinical Oncology, vol. 14, no. 9, pp. 531-548, 2017.

[29] B. Yang, W. Y. Xiong, and H. J. Hou, "Exosomal miRNAs as biomarkers of cancer: a meta-analysis," Clinical Laboratory, vol. 65, no. 5, 2019.

[30] J. Zhou, H. Guo, Y. Yang, Y. Zhang, and H. Liu, "A metaanalysis on the prognosis of exosomal miRNAs in all solid tumor patients," Medicine (Baltimore), vol. 98, no. 16, Article ID e15335, 2019.

[31] O. Hilly, N. Pillar, S. Stern et al., "Distinctive pattern of let-7 family microRNAs in aggressive carcinoma of the oral tongue in young patients," Oncology Letters, vol. 12, no. 3, pp. 1729-1736, 2016.

[32] R. Yang, Y. Shui, S. Hu, K. Zhang, Y. Wang, and Y. Peng, "Silenced myeloblastosis protein suppresses oral tongue squamous cell carcinoma via the microRNA-130a/Cylindromatosis axis," Cancer Management and Research, vol. 12, pp. 6935-6946, 2020.

[33] J. Chen, D. Yan, W. Wu, J. Zhu, W. Ye, and Q. Shu, "MicroRNA-130a promotes the metastasis and epithelialmesenchymal transition of osteosarcoma by targeting PTEN," Oncology Reports, vol. 35, no. 6, pp. 3285-3292, 2016.
[34] K. Asukai, K. Kawamoto, H. Eguchi et al., "Micro-RNA-130a-3p regulates gemcitabine resistance via PPARG in cholangiocarcinoma," Annals of Surgical Oncology, vol. 24, no. 8, pp. 2344-2352, 2017.

[35] Y. Zhou, R. Li, H. Yu, R. Wang, and Z. Shen, "microRNA$130 \mathrm{a}$ is an oncomir suppressing the expression of CRMP4 in gastric cancer," OncoTargets and Therapy, vol. 10, pp. 38933905, 2017.

[36] N. Suenaga, M. Kuramitsu, K. Komure et al., "Loss of tumor suppressor CYLD expression triggers cisplatin resistance in oral squamous cell carcinoma," International Journal of Molecular Sciences, vol. 20, no. 20, p. 5194, 2019.

[37] R. Massoumi, "CYLD: a deubiquitination enzyme with multiple roles in cancer," Future Oncology, vol. 7, no. 2, pp. 285-297, 2011.

[38] H. Jiang, W.-W. Yu, L.-L. Wang, and Y. Peng, "miR-130a acts as a potential diagnostic biomarker and promotes gastric cancer migration, invasion and proliferation by targeting RUNX3," Oncology Reports, vol. 34, no. 3, pp. 1153-1161, 2015.

[39] Z. Peng, F. Duan, J. Yin, Y. Feng, Z. Yang, and J. Shang, "Prognostic values of microRNA-130 family expression in patients with cancer: a meta-analysis and database test," Journal of Translational Medicine, vol. 17, no. 1, p. 347, 2019. 